C. Bernbeck, Freie Fettsäure als Ursache d. Ungeniessbark. v. Weizenmehl. 337

\title{
Freie Fettsäure als Ursache der Ungeniessbarkeit von Weizenmehl.
}

Von C. Berubeck in Germersheim.

Der vorliegende Fall dürfte, seiner Eigenartigkeit wegen, von allgemeinem Interesse sein und übergebe ich deshalb das in einer Streitsache abgegebene Gutachten in nachstehender Weise der Oeffentlichkeit.

Am 26. Januar 1881 erhielt der Unterzeichnete eine Kiloprobe eines angeblich gefälschten oder verdorbenen Mehles zur chemischen und physicalischen Untersuchung, welche folgendes Resultat lieferte:

Das betreffende Mehl hatte die Farbe des im Handel mit secunda bezeichneten Productes, schmeckte, auf die Zunge gebracht und im Munde langsam zertheilt, anfangs mild weizenartig, später jedoch bitterlich, im Schlunde lang anhaltend kratzend. Der Geruch war dumpfig, undefinirbar widerlich.

Unter der Loupe wurde nichts Bemerkenswerthes wahrgenommen, d. h. es fanden sich weder Mehlwürmer, Milben, noch andere niedere Thiere, die gewöhnlichen Begleiter verdorbenen Mehles. Unter dem Mikroskope konnten nur Stärkemehlkörner des Weizens beobachtet werden, dann noch wesentliche Mengen von Kleie, an denen ganz vereinzelte Spuren von Schimmel wahrzunehmen waren, Fettzellen und Kleberzellen. Mithin ergab der mikroskopische Befund unverfälschtes Weizenmehl 2. Qualität mit unwesentlichen Schimmelspuren.

Der chemische Befund war folgender:

Zur Vorprüfung wurde das Mehl erhitzt, wobei sich ein penetranter, fettartiger Geruch entwickelte; mit Wasser 1 : 5 angerührt, röthete dieses Gemenge sofort ein damit in innige Berührung gebrachtes Stückchen blaues Lackmuspapier, sichere Anzeichen einer vorhandenen Säure und war es hiebei auffallend, dass das mit dem Mehle in Berührung gewesene destillirte Wasser vollständig neutrale Reaction zeigte; mithin war die unzweifelhaft constatirte Säure in der Reihe derjenigen zu suchen, welche in Wasser unlöslich sind. Als nächste Lösungssnittel wurden alsdann absoluter Alkohol und Aether angewandt, welche beide, nach Abfiltration vom Mehle, intensiv saure Reaction (besonders der Aetherauszug) zeigten. Zur Feststellung der Identität dieser Säure, Arch, d Pharm. XVIII, Bds, 5, Heft. 
welche hier ohne Zweifel die wesentlichste Rolle spielt, wurde der Aetherauszug verdunstet, wobei ein klares, gelbes Fett, ganz übereinstimmend mit dem Weizenfette, gewonnen wurde, welches jedoch genau denselben unangenehmen Geschmack und Geruch des Mehles in concentrirtester Form zeigte und auf blaues Lackmuspapier stark röthend wirkte.

Mit Normalalkali bis zur alkalischen Reaction versetzt, liess sich das Fett klar verseifen. Die gebildete Seife löste sich fast klar in Wasser.

Somit war kein Zweifel mehr vorhanden, dass die beobachtete saure Reaction von einer freien Fettsäure, welche sich durch Zersetzung des Weizenfettes gebildet, herrührte und wie ich weiter unten durch practische Versuche beweisen werde, die Ursache aller unangenehmen Eigenschaften des Mehles war.

Die Resultate der Bestimmungen des Wassergehaltes der Asche, des Klebers und des Fettgehaltes sind in nachstehender $\mathrm{Zu}$ sammenstellung ausged rückt:

$$
\begin{aligned}
& \text { Wassergehalt . . . . . . . } 12,9 \% \\
& \text { Asche . . . . . . . . . } 1,1 \text { - } \\
& \text { Kleber . . . . . . . . . } 32,0 \text { - } \\
& \text { Fett mit freier Fettsäure - . 1 1,65 - } \\
& \text { Weizenstärke u. Zellsubstanz (Kleie) } 52,35-\frac{1}{\text { in } 100 \text { Theilen. }}
\end{aligned}
$$

Das specifische Gewicht des bei $100^{\circ} \mathrm{C}$. getrockneten Mehles, nach der Dietsch'schen Methode bestimmt, war 0,619 , mithin unverfälschtem und unverändertem Weizenmehle entsprechend.

Bei obigen Zahlen sind absolut normal der Wassergehalt und Kleber, etwas hoch, jedoch einem Weizenmehle 2. oder 3. Sorte noch entsprechend, die für Asche und Fett, indem dieselben durch höheren Gehalt des Mehles an Kleien bedingt sind; zu bemerken wäre hier noch, dass der kalt bereitete Aetherauszug des Mehles viel milder schmeckte, als der warm bei $40^{\circ} \mathrm{O}$. bereitete; ein Beweis, dass die verharzten, fettsäurereichsten Theile des hier vorhandenen Weizenfettes in der Wärme vollständiger gelöst wurden.

Die mit dem Mehle angestellten Backversuche ergaben Folgendes:

Das Mehl war im Stande, analog gutem Weizenmehle, $50 \%$ seines Gewichtes an Wasser zu binden, verhielt sich beim Verarbeiten zu Teig, Versetzen mit Hefe und Backen ganz conform 
mit Ausnahme des unangenehmen dumpfigen Geruches und Geschmackes nach ranzigem Fette, der sich durch die Hitze des Backofens, wie dies nicht anders zu erwarten, dem fertigen Brode in noch höherem Grade mittheilte, dasselbe beim Genusse eckelerregend machte.

Ein weiterer Backversuch durch Versetzen mit $1 / 1500$ Alaun führte zu keinem Resultate, indem sich Geruch und Geschmack ganz gleich, wie oben beschrieben, verhielt.

Nach dem Vorstehenden kann kein Zweifel darïber besteben, dass die Ursache der Ungeniessbarkeit des fraglichen Mehles durch seinen Gehalt an ranzigem Fette bedingt wurde; eine Annahme, die durch den Gegenversuch, der durch Versetzen eines aus reinstem Weizenmehle bereiteten Teiges mit 1/4\% Oelsäure ein Gebäck von gleichem Geruch und Geschmack bildete, zur Gewissheit wurde.

Die Frage, woher kommt die Rancidität des natürlichen Fettes des Weizens, welche Zeitdauer ist nöthig, um eine derartige Zersetzung möglich zu machen, wirft sich jetzt von selbst auf und soll deren Beantwortung im Nachstehenden versucht werden.

Die Hülle (Pericarpium) des Weizens besteht aus 3 mit einander verwachsenen Schichten, von welchen die beiden inneren reich an Stickstoffverbindungen, Phosphaten und Fett sind. Sie bilden gleichsam die von der Natur gebildete und weise zusammengesetzte Schutzhülle des inneren weissen Kernes, welcher vorzugsweise aus Stärkemehl besteht.

Da das fragliche Mehl in seiner sonstigen Zusammensetzung normal ist, so dürfte mit ziemlicher Gewissheit angenommen werden, dass die constatirte Umsetzung des Weizenfettes, schon in der Frucht selbst, vor dem Verarbeiten zu Mehl, sei es durch Einwirkung der Atmosphärilien, Alter des Weizens oder mangelhafte Ernte u. s. w., begonnen hat.

Es dürfte diese Annahme um so grössere Berechtigung haben, da das Fett (durch verschiedene Versuche beobachtet) gleichmässig in dem Mehle vertheilt ist und das Mehl aus allen Theilen entnommen gleich sauer reagirt. Wäre das Mehl tadellos versandt worden, so müssten die Umstände, die das Ranzidwerden des Fettes desselben (Feuchtigkeit, Wärme u. s. w.) bedingen, auch die übrigen Bestandtheile wie Stärkemehl, stickstoffhaltige Körper alteriren. Es ist dies jedoch in keiner Weise nachweisbar und 
muss deshalb die Ursache grösstentheils auf das ursprüngliche Mehlproduct zurückgeführt werden. Bemerkt muss hier noch werden, dass das mit Aether behandelte Mehl nicht mehr sauer reagirt und die Eigenschaften eines guten, geruchlosen Productes annimmt, ein schlagender Beweis, dass die nach Abdunsten des Aethers verbleibende fettsäurereiche Masse die Ursache der Ungeniessbarkeit in sich schliesst. Die Annahme, dass das aus dem betreffenden Weizen hergestellte Blumenmehl, da hier Kleientheile (besonders beim Mahlen mit Walzen) in hohem Grade ausgeschlossen sind, nur sehr wenig oder unmerklich von jenem ranciden Fette aufgenommen, liegt sehr nahe; letzteres concentrirt sich mehr in den Endproducten des Mahlprocesses, dem Nachmehle und der Kleie.

Schliesslich resumirte ich dahin, dass die Ursache der verwerflichen Eigenschaft jenes Mehles lediglich in dem Gehalte an freien Fettsäuren (rancidem Weizenfette) zu suchen ist; dass zur Bildung derselben besondere Zufälle und längere Zeit erforderlich sind; dass ein derartiges Mehl durch die Wirkung der Fettsäuren auf die Schleimhäute des Schlundes, Magens u. s. w. ekelerregend und gesundheitsschädlich wirken muss und deshalb für Zwecke der Ernährung für Nenschen unbrauchbar ist.

\section{Das Wasserlein'sche Saccharimeter.}

Von M. Schlesinger, Apotheker in Grünberg i/Sehl.

Gelegentlich einiger Harnanalysen fiel es mir auf, dass die Angaben des Saccharimeters ganz und gar nicht mit den Resultaten der chemischen Analyse übereinstimmten. Anfänglich glaubte ich, verleitet durch die niedrigen Gehalts - Angaben des Saccharimeters, dass der betreffende Harn vielleicht neben Dextrose auch Invertzucker enthielte, indessen widersprachen dieser Annahme die zu diesem Zweck vorgenommenen Titrirungen mit JodkaliumJodquecksilber-Lösung. Dies veranlasste mich endlich, das Instrument selbst einer eingehenden Prüfung zu unterziehen; hervorheben will ich noch, dass ich mittlerweile auch eine kurze Notiz im Archiv von Erdmann fand, welcher das Instrument für unbrauchbar erklärte. Die Kreiseintheilung ist zunächst nicht in 360 Grade, sondern in 